\title{
Influence des conditions d'alimentation hydrique sur le débourrement et la croissance de jeunes plants de Cèdres (Cedrus atlantica Manetti) cultivés en serre
}

\author{
D. FINKELSTEIN \\ I.N.R.A., Station de Sylviculture et de Production \\ Centre de Recherches forestières de Nancy, \\ Champenoux, F 54280 Seichamps
}

\section{Résumé}

- Deux expériences sur le cèdre réalisées en serre ont permis d'étudier l'effet de stress hydriques plus ou moins prononcés sur la phénologie des plants : débourrement et croissance en hauteur.

- La première expérience, portant sur des plants de la provenance Ventoux. âgés de 4 ans, a débuté en 1977 et s'est poursuivie jusqu'au printemps 1980. Pendant cette période certains plants ont été constamment bien alimentés en eau et d'autres ont subi diverses séquences hydriques : année sèche - année humide - année sèche par exemple.

Le déficit hydrique a tout d'abord un effet l'année même de son établissement : i] induit une baisse de la vitesse de croissance et un arrêt précoce de l'élongation. Il en résulte une diminution notable de la longueur finale de la pousse annuelle.

Mais l'influence du stress hydrique se manifeste également l'année suivante : les plants ayant subi une séquence année sèche - année humide montrent, la deuxième année, une élongation supérieure à celle des plants maintenus durant les 2 ans en conditions hydriques optimales. Ce phénomène est dû à un débourrement plus précoce des plants stressés et à une stimulation de leur vitesse de croissance.

- La seconde expérience a été réalisée sur 5 provenances de cèdres. Elle confirme l'ensemble des résultats décrits ci-dessus en montrant de plus des variations importantes dans l'intensité de la réaction à un stress hydrique donné suivant les provenances.

Ces différences pourraient être mises à profit pour orienter le choix des provenances vers les zones climatiques à moindre risque (sécheresse et gel tardif) pour chacune d'entre elles.

\section{1. - Introduction}

S'il est vrai que le rôle du cèdre en région méditerranéenne française fut tout d'abord de protection du sol et d'esthétisme, il est maintenant acquis qu'au niveau de la production de bois, cette essence est parfaitement compétitive tant vis-à-vis des espèces autochtones (ТоTH, 1971, 1973 ; B.V.F., 1974 b) que des essences intro- 
duites (pins noirs en particulier). Il en résulte un intérêt grandissant des sylviculteurs de cette région pour le cèdre et la nécessité de cerner avec précision tous les facteurs de sa croissance. Des études de terrain ont déjà été entreprises en Provence-Côte d'Azur et en Languedoc-Roussillon (YI, 1976 ; B.V.F., 1974 a, 1979). Mais linteraction des différentes variables climatiques ou édaphiques limite liinterprétation du comportement écologique. Il est donc souhaitable de compléter ces travaux par des études en conditions contrôlées qui permettent de tester l'influence individuelle des principaux paramètres climatiques.

C'est dans cette optique qu'ont été mises en place, en serre à Nancy, deux expériences sur le cèdre dans le but d'étudier l'effet de stress hydriques plus ou moins prononcés sur les phases phénologiques successives (débourrement et croissance en hauteur). Ces expérimentations ont été installées par AussenaC G. en 1976 et 1978 .

Il convient de préciser qu'il ne s'agit ici que d'une analyse préliminaire de ces deux expériences. Aucune analyse statistique poussée n’a encore été faite. D`autre part, certaines données non encore exploitées préciseront ultérieurement d'autres aspects du problème (biomasse, transpiration).

\section{2. - Matériel et méthodes}

\section{1. - Généralités communes aux deux expériences}

Les plants retenus ont été choisis parmi un échantillon moyen d'un lot plus important dont on avait éliminé les extrêmes (arbres les plus vigoureux et les plus chétifs).

Le substrat utilisé provient d'un horizon $A^{1}$ des sols bruns lessivés de texture limono-argileuse que l'on rencontre en forêt domaniale d'Amance (Meurthe-etMoselle). La relation $\mathrm{pF} /$ humidité a été établie pour cette terre, permettant ainsi de maintenir les plants à la capacité au champ ou tout autre niveau d'humidité désiré, en connaissant simplement le poids de la terre utilisée dans le pot.

Les deux expériences se sont déroulées dans une serre dite «froide», le chauffage éventuel durant l'hiver n'assurant que le maintien hors-gel des diverses installations. En été, la température est régulée par l'ouverture de auvents intervenant à $20^{\circ} \mathrm{C}$ et à partir de $25^{\circ} \mathrm{C}$ par un système de refroidissement par évaporation d'eau.

- Pour quantifier les différentes phases de débourrement nous avons adopté l'échelle de notation phénologique définie par Debazac (1965, in Aussenac, 1975) :

- 0 (b 0) : bourgeon non gonflé ayant conservé ses dimensions hivernales;

- 1 (b 1) : bourgeon gonflé présentant des écailles plus ou moins écartées ;

- 2 (b 2) : la jeune pousse apparaît par transparence mais l'enveloppe protectrice n'est pas encore éclatée ; 
- 3 (b 3) : bourgeon éclaté. Aiguilles de la pousse partiellement libérées. Le débourrement est effectif ;

- 4 (b 4) : jeune pousse entièrement libérée. Début de la phase de croissance.

Au moment où la croissance devient mesurable, on parle de stade b 4 mesurable.

Le stade réel de débourrement se situe entre le stade b 2 et le stade b 3 (Aussenac, 1975). Nous avons alors défini la «date de débourrement» d'un traitement donné comme étant celle pour laquelle la moyenne des notes attribuée à chaque plant de ce traitement est égale à 2,5 .

- La durée de saison de végétation correspond au temps (exprimé en nombre de jours) compris entre la date de débourrement définie ci-dessus et la date de fin de croissance.

- Enfin, nous avons été amenés à considérer la vitesse de croissance (expriméc en $\mathrm{mm} /$ jour) durant la période de croissance maximum. Nous l'avons estimée au vu des courbes comme le rapport de la longueur de la pousse à 90 p. 100 de la croissance totale sur la durée de la période correspondante.

\section{2. - Effets de régimes hydriques différents sur des plants de même provenance.}

\subsection{Origine des plants}

Cette expérience a été réalisée uniquement sur la provenance Mont-Ventoux (Vaucluse) de Cedrus atlantica Manetti. 180 plants ont été repiqués début mars 1976 à l'âge de 4 ans dans des pots en plastique d'une contenance de 9 litres.

\subsection{Protocole expérimental}

Dans chaque pot, un tuyau plastique percé sur toute sa longueur a été placé en spirale afin d'assurer une répartition homogène de l'eau lors des restitutions ultérieures.

\section{Année 1977}

Au début de 1977, les arbres tirés au hasard, ont été répartis en trois modalités (60 plants par modalité) :

- modalité humide : arbres maintenus à un niveau d'humidité pondérale de 32 p. 100 (pF 2,4 env.);

- modalité sèche : arbres maintenus à un niveau d'humidité pondérale de 16 p. 100 (pF 3,6 env.);

- modalité alternative : arbres subissant des cycles de dessèchement jusqu'à 16 p. 100 et de réalimentation à 32 p. 100.

Les restitutions sont effectuées à raison de 2 par semaine par pesée individuelle des pots sur une balance électrique (portée $13 \mathrm{~kg}$, précision $1 \mathrm{~g}$ ) et, si besoin est, réhydratation jusqu'au niveau d'humidité du sol désiré. 
L'application du dessèchement a débuté lorsque tous les plants ont atteint le stade phénologique de débourrement b 4 mesurable.

Les observations phénologiques (débourrement puis élongation de la pousse terminale) ont lieu 2 fois par semaine en début de saison puis 1 fois seulement lorsque la vitesse d'élongation diminue. En fin de saison, il est effectué sur chaque plant une mesure de hauteur finale 'de la pousse annuelle.

Ces modalités ont été appliquées jusqu'au 30 octobre. A partir de cette date, l'ensemble des plants a été restitué régulièrement à $32 \mathrm{p} .100$ durant tout l'hiver.

\section{Année 1978}

Chaque modalité 1977 a été divisée en trois nouvelles modalités humide, sèche et alternative de telle sorte que nous disposons pour cette année de 9 traitements (20 plants par modalité). Les protocoles de restitution et de mesures sont identiques à ceux adoptés pour 1977.

Les plants ont tous été restitués à 32 p. 100 à dater du 13 octobre.

\section{Année 1979}

Les neuf traitements ont été à nouveau scindés mais cette fois de façon dichotomique (humide et alternatif), car trois nouvelles modalités auraient trop réduit le nombre d'individus de chacune d'entre elles et les résultats auraient été difficiles à interpréter. Il reste donc 10 plants pour chacune des 18 modalités.

De plus, le protocole des restitutions a été simplifié. Chaque pot a été percé à sa base et l'arrosage s'effectue directement au jet, sans pesée, à raison de 2 fois par semaine pour le traitement humide et 1 fois par quinzaine pour le traitement alternatif.

Comme les deux années précédentes, les arbres ont été ramenés à la capacité au champ le 10 octobre.

Le schéma définitif de ces trois années d'expérience est donc le suivant :

\begin{tabular}{|c|c|c|c|c|c|c|c|c|c|c|c|c|c|c|c|c|c|}
\hline 1977 & \multicolumn{6}{|c|}{$32 \%$} & \multicolumn{6}{|c|}{$16 \% \rightleftharpoons 32 \%$} & \multicolumn{5}{|c|}{$16 \%$} \\
\hline 1978 & \multicolumn{2}{|c|}{32} & \multicolumn{2}{|c|}{$\rightleftharpoons$} & \multicolumn{2}{|c|}{16} & \multicolumn{2}{|c|}{32} & \multicolumn{2}{|c|}{$\rightleftharpoons$} & \multicolumn{2}{|c|}{16} & \multicolumn{2}{|c|}{32} & \multicolumn{2}{|c|}{$\rightleftharpoons$} & 16 \\
\hline 1979 & 32 & $\rightleftharpoons$ & 32 & $\rightleftharpoons$ & 32 & $\rightleftharpoons$ & 32 & $\rightleftharpoons$ & 32 & $\rightleftharpoons$ & 32 & $\rightleftharpoons$ & 32 & $\rightleftharpoons$ & 32 & $\rightleftharpoons$ & 32 \\
\hline
\end{tabular}

Le symbole $\rightleftharpoons$ correspond au traitement $16 \% \rightleftharpoons 32 \%$.

\section{3. - Etude comparative des effets de la sécheresse} donnée sur des plants de provenances différentes

\subsection{Origine des plants}

Cinq provenances de Cedrus atlantica Manetti ont été utilisées pour cette expérience : 
- Talarine (Maroc, Moyen-Atlas)

Symbole Tari

- Babors (Algérie)

Babo

- Chélia (Algérie, Aurès)

Chel

- Rialsesse (France)

Rial

- Lubéron (France)

Lube

ainsi qu'une provenance de Cedrus libani (Loudon) :

- Aslankoy (Turquie)

Asla

18 plants ont été retenus pour Tari, Chel, Rial et Lube, 12 pour Babo et 10 pour Asla.

Les plants ont été repiqués début février 1978 à l'âge de trois ans, dans des containers plastiques d'une contenance de 50 litres, à raison de 6 plants par container (5 pour Asla).

\subsection{Protocole expérimental}

\section{Année 1978}

Les containers ne sont pas percés.

Aucun dessèchement n'a été conduit durant la saison de végétation. Chaque container est réalimenté à la capacité au champ une fois par semaine, par pesée sur une balance mécanique de précision (portée $100 \mathrm{~kg}$, précision $50 \mathrm{~g}$ ).

\section{Année 1979}

Les containers ont cette fois été percés à leur base. Comme précédemment, les modalités de dessèchement n'ont été appliquées qu'à partir de la date à laquelle tous les plants ont atteint le stade phénologique b 4 mesurable.

Pour les provenances comprenant 18 plants ( 3 containers), 3 modalités ont été mises en place :

— traitement «humide» : arrosage 2 fois par semaine ;

— traitement $\ll \sec » \quad:$ arrosage 1 fois par semaine ;

— traitement «très sec» : arrosage 1 fois par quinzaine.

Les provenances ne comprenant que 12 plants ( 2 containers) n'ont été soumises qu'aux traitements «humide» et «très $\sec »$.

Malgré le perçage des containers, le traitement «humide» de la provenance Aslankoy a subi un engorgement et trois plants sur cinq sont morts au cours de la saison de végétation. Ce traitement ne pourra donc être pris en compte dans les résultats de cette expérience.

Durant les deux années d'expérience, des mesures hebdomadaires d'élongation des pousses terminales ont été effectuées. 


\section{3. - Résultats}

\section{1. - Première expérience (provenance Ventoux)}

\subsection{Croissance en hauteur}

Les différentes modalités ont tout d'abord un effet immédiat sur l'élongation de la pousse annuelle. La figure 1 fournit en exemple les différences observées entre les trois modalités de 1977. Nous voyons ainsi qu'un stress hydrique élevé entraîne une vitesse de croissance réduite (pente de la courbe) et d'autre part un arrêt précoce de l'élongation. Il en résulte une diminution très notable de la longueur finale de la pousse annuelle. Le tableau 1 donne les croissances moyennes calculées par modalité et par année, ainsi que les dates moyennes de fin de croissance.

\section{Tableau 1}

Influence des modalités sur la date de fin de croissance et la longueur finale de la pousse terminale

Influence of modalities on date of termination of growth and final length of terminal shoot

\begin{tabular}{|c|c|c|c|}
\hline \multirow{2}{*}{ Année $n$} & \multicolumn{3}{|c|}{ Modalités $\mathrm{n}$} \\
\hline & $32 \%$ & $16 \% \rightleftharpoons 32 \%$ & $16 \%$ \\
\hline \multicolumn{4}{|l|}{1977} \\
\hline Date $\ldots \ldots \ldots \ldots \ldots$ & $16 / 08$ & $01 / 08$ & $27 / 06$ \\
\hline Longueur $\ldots \ldots \ldots \ldots$ & $218 \mathrm{~mm}$ & $112 \mathrm{~mm}$ & $63 \mathrm{~mm}$ \\
\hline \multicolumn{4}{|l|}{1978} \\
\hline Date $\ldots \ldots \ldots \ldots \ldots \ldots$ & $22 / 08$ & $13 / 07$ & $23 / 06$ \\
\hline Longueur $\ldots \ldots \ldots \ldots \ldots$ & $147 \mathrm{~mm}$ & $69 \mathrm{~mm}$ & $47 \mathrm{~mm}$ \\
\hline \multicolumn{4}{|l|}{1979} \\
\hline Date $\ldots$. & $16 / 08$ & $15 / 06$ & - \\
\hline Longueur & $142 \mathrm{~mm}$ & $39 \mathrm{~mm}$ & \\
\hline
\end{tabular}

L'effet des modalités de l'année n se fait également sentir sur la croissance de l'année $\mathbf{n}+1$. Comparons à cet égard les plants des 3 traitements différents en 1977 et soumis à la même modalité (sèche en l'occurrence) en 1978 (fig. 2). Les arbres ayant subi les dessèchements les plus prononcés en 1977, ont en 1978 la vitesse de croissance la plus élevée et présentent durant la saison 1978 une élongation annuelle 
nettement supérieure à celle des plants ayant été constamment alimentés en eau durant les 2 saisons.

Le phénomène est général puisqu'il en va ainsi durant les trois années de l'expérimentation pour les diverses séquences d’alimentation hydrique (tableaux 2 et 3 ).

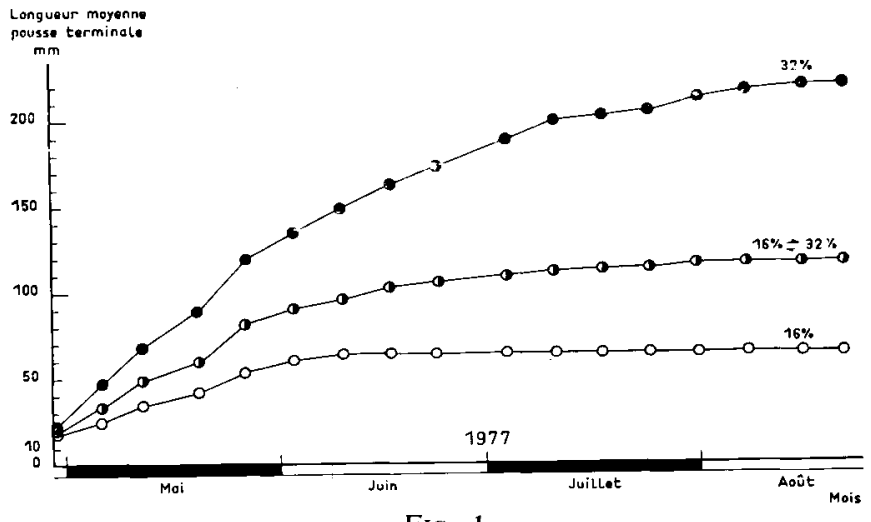

FIG. 1

Evolution de la longueur moyenne de la pousse terminale en 1977 pour les 3 traitements (32 p. 100 : capacité au champ, 16 p. 100 sécheresse, $16 \rightleftarrows 32$ p. 100 : cycle humidité-sécheresse)

Course of the mean length of terminal shoot in 1977 for the 3 modalities (32 p. 100 : field capacity, 16 p. 100 drought, 16 p. $100 \rightleftarrows 32$ p. 100 : cycle moisture-drought)

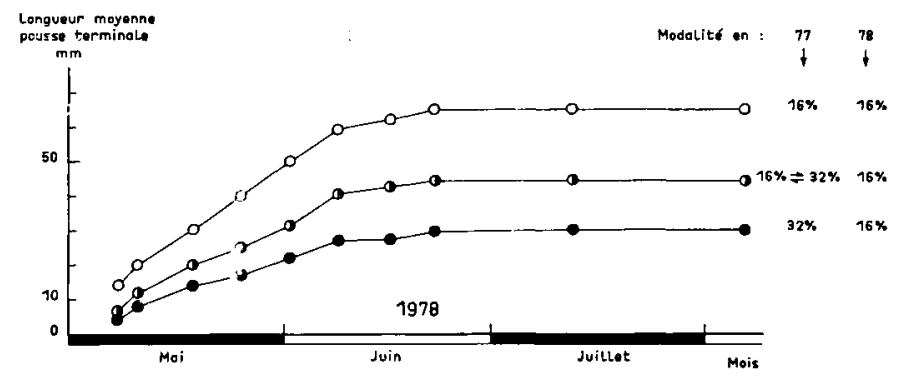

Fig. 2

Evolution de la longueur moyenne de la pousse terminale 1978 des 3 traitements soumis à lu modalité 16 p. 100 (sécheresse) en 1978

Course of the mean lenght of 1978 terminal shoot for the 3 treatments grown in 16 p. 100 modality (drought) in 1978 
D. FINKELSTEIN

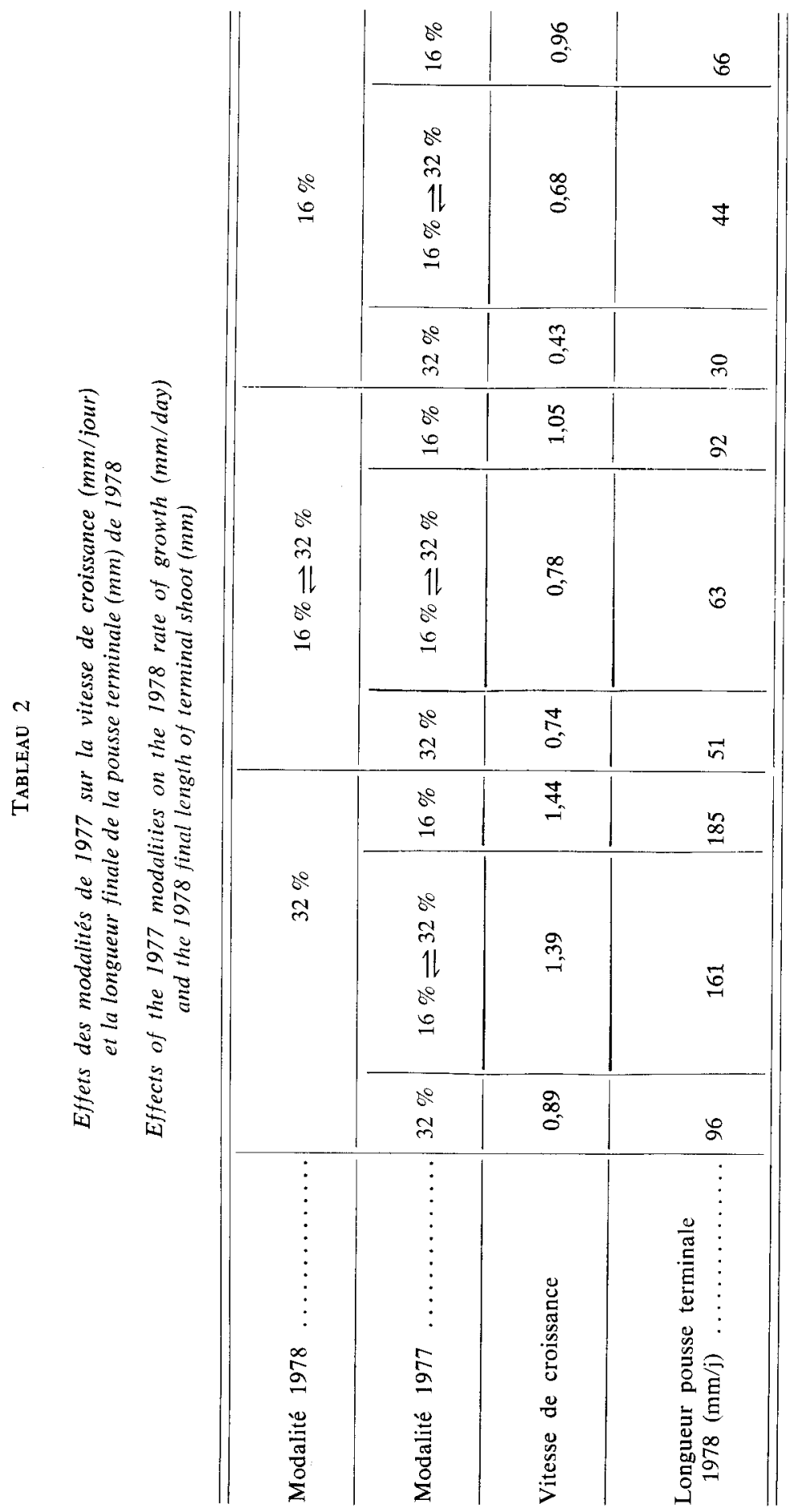


Tablead 3

Effets des modalités 1978 sur la vitesse de croissance ( $\mathrm{mm} /$ jour) et la longueur finale de la pousse terminale $(\mathrm{mm})$ de 1979

Effects of the 1978 modalities on the 1979 rate of growth (mm/day) and the 1979 final length of terminal shoot ( $\mathrm{mm}$ )

\begin{tabular}{|c|c|c|c|c|c|c|}
\hline \multirow{2}{*}{$\frac{\text { Modalité } 1979 \ldots \ldots \ldots}{\text { Modalité } 1978 \ldots \ldots \ldots}$} & \multicolumn{3}{|c|}{$32 \%$} & \multicolumn{3}{|c|}{$16 \% \rightleftharpoons 32 \%$} \\
\hline & $32 \%$ & $16 \% \rightleftharpoons 32 \%$ & $16 \%$ & $32 \%$ & $16 \% \rightleftharpoons 32 \%$ & $16 \%$ \\
\hline Vitesse de crissence $(\mathrm{mm} / \mathrm{j})$ & 0,91 & 1,45 & 1,47 & 0,29 & 0,59 & 0,70 \\
\hline $\begin{array}{r}\text { Longueur pousse terminale } \\
1979(\mathrm{~mm}) \ldots \ldots \ldots \ldots\end{array}$ & 93 & 159 & 181 & 20 & 45 & 59 \\
\hline
\end{tabular}

\subsection{Débourrement}

L'effet des traitements se manifeste également au printemps suivant par un débourrement d'autant plus précoce que les plants ont subi un stress hydrique plus important (tableau 4).

\section{TABLEAU 4}

Effet des modalités de l'année $n$ sur la précocité du débourrement de l'année $n+1$

Effect of the n-year modalities on the precocity of the $(n+1)$-year budbreak

\begin{tabular}{|c|c|c|c|}
\hline \multirow{2}{*}{ Année $\mathrm{n}+1$} & \multirow{2}{*}{$32 \%$} & \multicolumn{2}{|l|}{ Modaiités n } \\
\hline & & $16 \% \rightleftharpoons 32 \%$ & $16 \%$ \\
\hline 1978 & $18 / 04$ & $13 / 04$ & $08 / 04$ \\
\hline 1979 & $17 / 04$ & $04 / 04$ & $24 / 03$ \\
\hline$\ldots \ldots \ldots \ldots \ldots \ldots$ & $05 / 04$ & $22 / 03$ & 一 \\
\hline
\end{tabular}

Mais nous pouvons voir également que pour un traitement donné la date de débourrement varie d'une année sur l'autre. Il existe donc des variations interannuelles indépendantes du stress hydrique. Il est très probable que la température des mois précédant l'éclatement des bourgeons joue un rôle prépondérant dans ces variations inter-annuelles. On sait en effet que le débourrement d'une espèce nécessite pour son avènement la satisfaction de ses besoins en froid et en chaleur, qui repré- 
sentent des constantes pour l'espèce considérée. Plusieurs types de calculs permettant l'estimation de ces constantes sont explicités par Brochet, Durand et Gerbier (1971) ainsi que par Bidabe (1967) : sommes de température et sommes de Q 10. AusseNAC (1975) a en particulier utilisé ces dernières pour étudier le débourrement de divers résineux. Malheureusement, aucune de ces méthodes n'a donné de résultats probants pour l'expérience présente. Le fait peut en être en grande partie attribué au manque d'informations : seulement trois années d’observations phénologiques.

L'influence certaine des températures printanières a néanmoins été mise en évidence en calculant pour chaque traitement de chaque année (30 points au total) la température moyenne de la période séparant le $1^{\text {er }}$ janvier et la date de débourrement du traitement.

Il a pu alors être calculé une régression linéaire multiple entre cette température moyenne, la date de fin de croissance de l'année $n$ (dont la précocité est le reflet de l'intensité du stress hydrique) et la date du débourrement de l'année $\mathbf{n}+1$ :

avec :

$$
\mathrm{z}=0,36 \mathrm{x}-3,14 \mathrm{y}+66,00 \quad \mathrm{r}^{2}=0,61
$$

$\mathrm{z}=$ date de débourrement $\mathrm{n}+1$, en nombre de jours depuis le $1^{\text {er }}$ janvier ;

$\mathrm{x}=$ date de fin de croissance $\mathrm{n}$, en nombre de jours depuis le $1^{\text {er }}$ janvier:

$\mathrm{y}=$ température moyenne définie ci-dessus, en degrés Celsius.

L'effet des modalités de l'année $\mathrm{n}$ sur le débourrement de l'année $\mathrm{n}+1$ se surimpose donc à l'influence de ces mêmes modalités sur la vitesse de croissance. Mais la part respective que prennent ces 2 paramètres dans les différences de longueur finale varie suivant les séquences d'alimentation hydrique.

En prenant comme référence la modalité déterminant le débourrement le plus tardif, on peut calculer l'avance de croissance prise au débourrement par les modalités plus précoces en début de saison de végétation. On calcule de la même manière la différence de longueur de pousse finale de ces 2 modalités par rapport à la modalité témoin qui permet alors d'établir le rapport de l'écart en début de saison de végétation à l'écart final (tableaux 5 et 6 ). Nous voyons que l'avance de croissance prise au débourrement par les modalités précoces peut expliquer jusqu'à 65 p. 100 de la différence des pousses finales.

\section{2. - Deuxième expérience (5 provenances)}

L'année 1978 devant être considérée comme une année préliminaire pendant laquelle les plants retrouvent un équilibre après leur crise de transplantation, les seuls résultats susceptibles d'être analysés concernent l'ensemble de l'année 1979 et le débourrement de 1980 .

\subsection{Débourrement (tableau 7)}

La comparaison des plants n'ayant pas subi de stress hydrique (1979 et 1980 , traitement humide) ne montre pas de classement constant des provenances d'une année sur l'autre. Toutes les provenances ont cependant en commun un débour- 
rement plus tardif en 1980 qu'en 1979 (de 30 jours plus tard pour Rialsesse à 53 pour Babors). Cette tendance va à l'encontre des résultats obtenus précédemment pour la provenance Ventoux (cf. 3.12) et ne confirme donc pas l'effet de la température au printemps, mais cette différence est difficile à interpréter, les deux expériences ayant beaucoup de points divergents (âge des plants, protocole...).

\section{TABleau 5}

Ecart de croissance (en $\mathrm{mm}$ ) des modalités précoces par rapport à la modalité la plus tardive (32 p. 100), en début et en fin de saison de végétation 1978

Difference of growth ( $\mathrm{mm}$ ) between the modality having the latest budbreak ( $32 \mathrm{p} .100)$ and the two earlier modalities, at the beginning and at the end of the 1978 growth season

\begin{tabular}{|c|c|c|c|c|}
\hline $\begin{array}{c}\text { Modalité } \\
1978\end{array}$ & $\begin{array}{c}\text { Modalité } \\
1977\end{array}$ & $\begin{array}{c}\text { Avance } \\
\text { de croissance } \\
\text { au } \\
\text { débourrement } \\
(\mathrm{mm})\end{array}$ & $\begin{array}{l}\text { Ecart } \\
\text { de croissance } \\
\text { en fin } \\
\text { de saison } \\
(\mathrm{mm})\end{array}$ & $\frac{\text { Avance début }}{\text { Ecart fin }} \times 100$ \\
\hline \multirow{3}{*}{$32 \%$} & $32 \%$ & 0 & 0 & - \\
\hline & $16 \% \rightleftharpoons 32 \%$ & +2 & +65 & $3,1 \%$ \\
\hline & $16 \%$ & +14 & +89 & $15,7 \%$ \\
\hline \multirow{3}{*}{$16 \%$} & $32 \%$ & 0 & 0 & - \\
\hline & $16 \% \rightleftharpoons 32 \%$ & 0 & +12 & $0 \%$ \\
\hline & $16 \%$ & +5 & +41 & $12,2 \%$ \\
\hline \multirow{3}{*}{$16 \% \rightleftharpoons 32 \%$} & $32 \%$ & 0 & 0 & - \\
\hline & $16 \% \rightleftharpoons 32 \%$ & +2 & +14 & $14,3 \%$ \\
\hline & $16 \%$ & +10 & +36 & $27,8 \%$ \\
\hline
\end{tabular}

Par contre, on retrouve nettement l'influence du dessèchement sur la date de débourrement de l'année suivante. En 1980, les traitements «très secs 1979 » ont tous débourré plus tôt que les «humides $1979 »$. Ne disposant que d'un nombre restreint de données par provenance, il n'est pas possible de quantifier plus précisément ce résultat, mais néanmoins il appuie de façon qualitative les observations de la première expérience. 


\section{TABLeau 6}

Ecart de croissance (en $\mathrm{mm}$ ) des modalités précoces par rapport à la modalité la plus tardive (32 p. 100), en début et en fin de saison de végétation 1979

Difference of growth ( $\mathrm{mm}$ ) between the modality having the latest budbreak ( $32 \mathrm{p.} 100)$ and the two earlier modalities at the beginning and at the end of the 1979 growth season

\begin{tabular}{|c|c|c|c|c|}
\hline $\begin{array}{l}\text { Modalité } \\
1979\end{array}$ & $\begin{array}{l}\text { Modalité } \\
1978\end{array}$ & $\begin{array}{c}\text { Avance } \\
\text { de croissance } \\
\text { au } \\
\text { débourrement } \\
(\mathrm{mm})\end{array}$ & $\begin{array}{c}\text { Ecart } \\
\text { de croissance } \\
\text { en fin } \\
\text { de saison } \\
(\mathrm{mm}) \\
\end{array}$ & $\frac{\text { Avance début }}{\text { Ecart fin }} \times 100$ \\
\hline \multirow{3}{*}{$32 \%$} & $32 \%$ & 0 & 0 & - \\
\hline & $16 \% \rightleftharpoons 32 \%$ & +13 & +66 & $19,2 \%$ \\
\hline & $16 \%$ & +30 & +88 & $34,4 \%$ \\
\hline \multirow{3}{*}{$16 \% \rightleftharpoons 32 \%$} & $32 \%$ & 0 & 0 & - \\
\hline & $16 \% \rightleftharpoons 32 \%$ & +11 & +25 & $44,0 \%$ \\
\hline & $16 \%$ & +25 & +39 & $64,9 \%$ \\
\hline
\end{tabular}

\section{TABLEAU 7}

Effet des modalités de l'année $n$ sur la date de débourrement de l'année $n+1$ pour les 5 provenances

Effect of the n-year modalities on the $(n+1)$-year budbreak date for the five provenances

\begin{tabular}{|c|c|c|c|}
\hline \multirow[b]{2}{*}{ Provenances } & \multirow{2}{*}{$\begin{array}{c}\text { Date } \\
\text { de débourrement } \\
1979 \\
\text { Modalité } \\
\text { humide } 1978\end{array}$} & \multicolumn{2}{|c|}{ Date de débourrement 1980} \\
\hline & & $\begin{array}{c}\text { Modalité } \\
\text { humide } 1979\end{array}$ & $\begin{array}{l}\text { Modalité } \\
\text { très sec } 1979\end{array}$ \\
\hline Lube & $11 / 03$ & $23 / 04$ & $28 / 03$ \\
\hline Rial $\ldots \ldots \ldots \ldots \ldots \ldots$ & $18 / 03$ & $26 / 04$ & $27 / 03$ \\
\hline Tari & $17 / 03$ & $25 / 04$ & $06 / 04$ \\
\hline Chel ........ & $09 / 03$ & $24 / 04$ & $27 / 03$ \\
\hline Babo & $02 / 03$ & $24 / 04$ & $28 / 02$ \\
\hline
\end{tabular}




\subsection{Durée de saison de végétation et croissance en hauteur}

La comparaison des rapports $\frac{\text { très secs }}{\text { humides }}$ pour la croissance et la saison de

végétation (tableau 8) montre que la sécheresse n’affecte pas ces deux paramètres avec la même intensité. Quelle que soit la provenance, la longueur de la pousse annuelle se voit beaucoup plus réduite que la saison de végétation. En d'autres termes, la sécheresse agit sur la durée de croissance mais également sur la vitesse de croissance.

\section{TABleau 8}

Effet des modalités « humide» et «très sec » sur la durée de saison de végétation et la croissance de la pousse terminale des 5 provenances pour l'année 1979

Effect of «well-watered» and «very droughty» modalities on duration of growth season and the growth of terminal shoot for the five provenances during 1979

\begin{tabular}{|c|c|c|c|c|c|c|}
\hline $\begin{array}{l}\text { Prove- } \\
\text { nances }\end{array}$ & 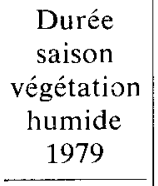 & $\begin{array}{c}\text { Durée } \\
\text { saison } \\
\text { végétation } \\
\text { très sec } \\
1979 \\
\end{array}$ & $\begin{array}{c}\begin{array}{c}\text { Rapport } \\
\text { saison }\end{array} \\
\frac{\text { très sec }}{\text { humide }} \times 100\end{array}$ & $\begin{array}{c}\text { Croissance } \\
\text { en hauteur } \\
\text { humide } \\
1979\end{array}$ & $\begin{array}{c}\text { Croissance } \\
\text { en hauteur } \\
\text { tris sec } \\
1979\end{array}$ & $\begin{array}{c}\begin{array}{c}\text { Rapport } \\
\text { croissance }\end{array} \\
\frac{\text { très sec }}{\text { humide }} \times 100 \\
\end{array}$ \\
\hline Lube & 111 jours & 90 jours & $81,1 \%$ & $277 \mathrm{~mm}$ & $122 \mathrm{~mm}$ & $44,0 \%$ \\
\hline Rial & 112 jours & 81 jours & $72,3 \%$ & $257 \mathrm{~mm}$ & $86 \mathrm{~mm}$ & $33,5 \%$ \\
\hline Tari & 115 jours & 80 jours & $69,6 \%$ & $291 \mathrm{~mm}$ & $114 \mathrm{~mm}$ & $39,2 \%$ \\
\hline Chel & 106 jours & 87 jours & $82,1 \%$ & $265 \mathrm{~mm}$ & $118 \mathrm{~mm}$ & $44,5 \%$ \\
\hline Babo & 107 jours & 100 jours & $93,5 \%$ & $231 \mathrm{~mm}$ & $162 \mathrm{~mm}$ & $70,1 \%$ \\
\hline
\end{tabular}

Dans ce cadre général, il faut souligner le comportement très intéressant de la provenance Babors dont la croissance et la durée de celle-ci ne sont que modérément touchées par la sécheresse (réduction respectivement de 30 p. 100 et 7 p. 100 par rapport à la modalité humide) ce qui lui permet ainsi d'arriver en tête des provenances en conditions de stress hydrique alors que son comportement était le plus médiocre en modalité humide.

\section{4. - Discussion}

Nous avons pu constater que les stress hydriques appliqués lors des deux expériences ont joué un rôle l'année même de leur application en réduisant notablement les pousses annuelles par rapport aux modalités humides. Le cèdre ayant 
la faculté de pousser tard dans l'année (octobre) lorsque les conditions environnementales (hydriques en particulier) sont favorables (Aussenac et al., 1980), il est certain qu'en conditions naturelles, un déficit hydrique aura également une influence importante sur la croissance en hauteur. ZAHNER (1968) signale plusieurs études allant dans ce sens.

En fait, l'effet du déficit sur la croissance d'une espèce dépend en grande partie de la cinétique de cette croissance. Ainsi que le montrent Clements (1970) sur Pinus resinosa et Becker (1974, 1977) sur Pseudotsuga menziesii, Abies alba, Abies nordmanniana et Abies numidica, on observe fréquemment chez les essences à pousse monocyclique courte, un décalage d'un an entre l'application de la sécheresse et les effets de celle-ci sur la croissance en hauteur. En revanche, les espèces à pousse monocyclique longue, ou polycyclique comme Pinus taeda (ZAHNer, 1962) montrent une réduction de leur croissance l'année même du stress hydrique. Le cèdre appartient donc à cette deuxième catégorie d'arbres.

Les deux expériences décrites ici ont également montré que la sécheresse d'une année donnée a une incidence sur la croissance de l'année suivante. Nous avons vu en effet que les plants soumis à un dessèchement (année $n$ ) ont, lorsqu'ils sont placés en conditions humides l'année suivante, une croissance $n+1$ supérieure à celle des plants maintenus durant les deux ans à la capacité au champ. Lors d'une succession d'années sèche puis humide, le «déficit de croissance » de la première année est donc presque intégralement compensé par une pousse plus importante la deuxième année. Une illustration en est fournie par la figure 3.

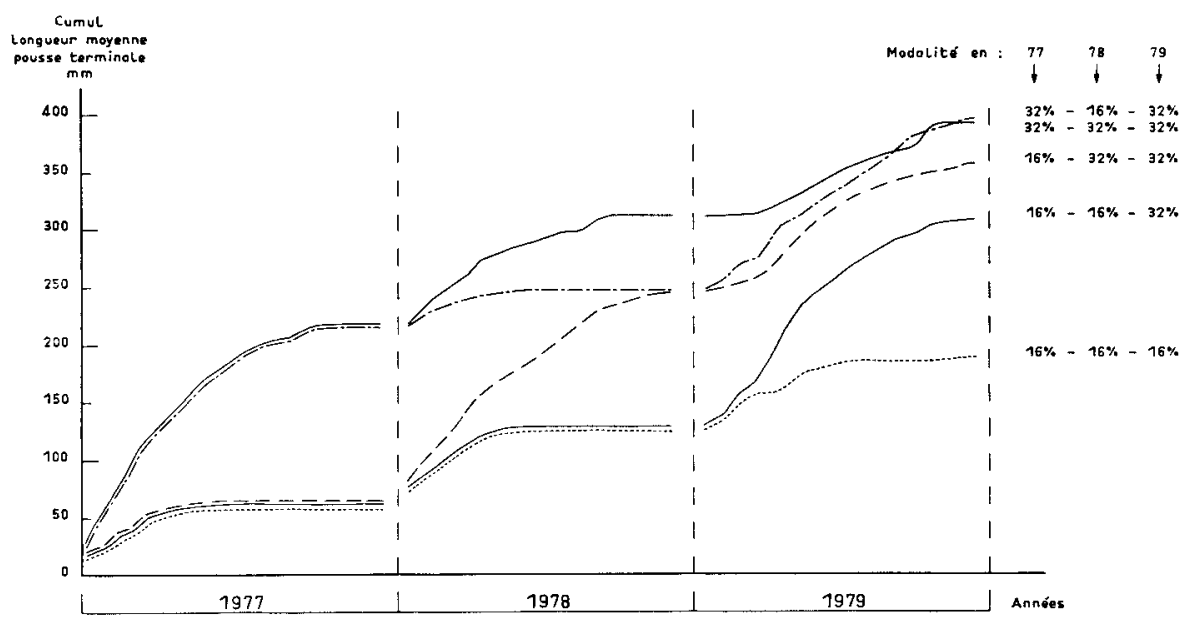

FIG. 3

Exemples de cumuls de longueur moyenne de la pousse terminale durant 3 années d'expérience (32 p. 100 : capacité au champ, 16 p. 100 : sécheresse) 
L'analyse complète des données a permis de lier dessèchement de l'année $\mathrm{n}$ et croissance de l'année $n+1$ : une sécheresse prononcée induit l'année suivante une vitesse de croissance plus élevée et un débourrement précoce. Nous avons vu que ce dernier peut tenir un rôle très important dans les différences de longueur finale des pousses terminales entre traitements.

Le problème du déterminisme du débourrement a surtout été étudié sous son aspect hormonal (Romberger, 1963; ZIMMERMANN \& Brown, 1971).

Mais comme le rappelle Kozlowskı (1971), le contrôle hormonal de la dormance et du débourrement ne joue qu'un rôle médiateur entre les fluctuations environnementales et les variations phénologiques observées.

Or à cet égard, si l'influence du photopériodisme et du thermopériodisme printanier ont principalement retenu l'attention, le rôle des conditions antérieures (sécheresse en particulier) n’est que peu évoqué dans la littérature, si ce n'est par son incidence sur la croissance (voir ci-dessus). Les travaux de Becker (1977) aboutissent par ailleurs à la conclusion qu'une sécheresse d'été entraîne un retard important au débourrement suivant. Ces résultats, bien que différents de ceux obtenus ici, ne sont pas nécessairement en contradiction si l'on souligne le fait que l'auteur travaillait sur des espèces à durée de croissance courte (Douglas, Sapins) dont les dates de fin de croissance peuvent être beaucoup moins dépendantes de la sécheresse qu'une essence dont la croissance se prolonge tard lorsque les conditions sont favorables. Par ailleurs, la sécheresse à laquelle furent soumises ces essences fut beaucoup plus intense $(\mathrm{pF}>4,2)$ et plus longue que celles opérées lors des expériences décrites ici. Or, Aussenac (1980), sur une expérience d'irrigation réalisée sur un jeune peuplement de douglas pendant la sécheresse exceptionnelle de 1976, a montré l'importance des déficits tardifs (septembre-octobre) sur la croissance de lannée suivante.

Le phénomène semble donc encore loin d'être généralisable. On peut toutefois, en le limitant au cas du cèdre, émettre quelques hypothèses quant à sa signification et ses conséquences pratiques. En particulier, le stress hydrique peut-il entraîner une réduction des besoins thermiques nécessaires au débourrement et donc du temps nécessaire à les satisfaire? Ou est-ce simplement l'arrêt précoce de la croissance qui fait que le bourgeon «comptabilise» plus tôt les actions froid puis les actions chaleur? On peut enfin mettre en avant une corrélation possible entre appareil racinaire et partie aérienne. En effet, daprès RIEDACKER (1978), si l'action du froid est une des conditions de levée de dormance, la présence de racines blanches pourrait jouer un rôle non négligeable en fournissant des produits de type gibberelline dont l'action est primordiale lors de la levée de dormance (SMITH \& KEFFORD, 1964). Il faut rapprocher cette remarque des travaux de VARTANian N. (1978 a, $1978 \mathrm{~b}$ ) sur Sinapis alba il est vrai : l'auteur y montre qu'en condition de sécheresse édaphique la rhizogénèse est stimulée. Les nouvelles racines formées ne sont susceptibles de s'allonger et d'acquérir un rôle fonctionnel qu'après réhumectation du sol. De plus, les plants soumis à l'assèchement puis réhumidifiés montrent une croissance racinaire supérieure à celle des plants témoins régulièrement arrosés durant toute leur croissance. Un tel processus pourrait alors expliquer un débourrement précoce par un apport plus important de gibberellines dû à une quantité supérieure de racines blanches qui pourraient également expliquer l'augmentation de la vitesse de croissance. 
Quel que soit le mécanisme fondamental incriminé, il est certain qu une date de débourrement précoce va entraîner des risques de gelées accrus. Or nous avons pu constater qu'un même stress hydrique provoquait une variation plus ou moins grande de la date de débourrement suivant les provenances. L'effet provenance est donc un facteur essentiel entrant en ligne de compte dans l'évaluation des risques de gelées. Actuellement peu d'informations sont disponibles à ce sujet. Des observations de débourrement effectuées dans l'Arboretum du Centre National de Recherches Forestières de Champenoux montrent peu de variations entre les provenances. Mais les stress subis ici par les arbres sont rarement conséquents et d'autres facteurs climatiques deviennent alors prépondérants. Seules des conditions sévères permettent de différencier les potentialités génétiques. Ainsi, au Treps dans le Massif des Maures, Pradal (1979) note en 1975 un débourrement très précoce des provenances orientales comparées aux provenances moyen-atlasiques, accompagné de dégâts de gelée très importants parmi les premières. Dans son aire d'origine le cèdre doit également faire face à des conditions climatiques extrêmes (sécheresse estivale, froid hivernal ou combinaison des deux) mais les différentes provenances sont supposées être en équilibre avec leur environnement et donc être adaptées aux risques engendrés par celui-ci.

Comparons par exemple les deux provenances algériennes dont nous disposions. La cédraie des Babors bénéficie en hiver de températures modérées alors que la provenance Chelia peut subir des froids très intenses (PradaL, 1979).

On peut constater sur le tableau 8 que la sécheresse qu'ont subie les plants en 1979 a fortement avancé le débourrement 1980 de la provenance ayant peu à craindre de gelées tardives (Babors : 55 jours d'avance pour les «très secs 1979 ») et plus modérément la provenance adaptée aux froids intenses et prolongés (Chélia : 28 jours d'avance pour les «très secs 1979 »).

Toutefois ces résultats sont trop peu étoffés et demandent à être confirmés.

Il conviendra donc de multiplier ces comparaisons de provenances dont PutoD (in B.V.F., 1979) souligne la nécessité pour le forestier de terrain. Mais s'ils se confirmaient, il y aurait là un critère intéressant de sélection qui permettrait d'orienter les provenances vers les zones climatiques leur convenant le mieux, en tenant compte des fréquences de fortes sécheresses et de gelées printanières.

Reçu pour publication en décembre 1980.

\section{Summary}

Influence of various water supply patterns on budbreak and height growth of young plants of Cedrus atlantica Manetti grown in greenhouse.

- Two experiments on Cedrus atlantica Manetti grown in greenhouse allowed to study the effect of more or less intense water stress on phenology : budbreak and height growth.

- The first experiment, using four-year-old plants originating from Mont-Ventoux (Vaucluse, France), has begun in 1977 and continued until spring 1980. During this period 
some plants were always remanied in optimal hydric conditions and others were subjected to various water supply : drought year-moist year-drought year for example.

Within the year of water deficit there is already a reduction of the rate of growth and an early stop of the elongation. It produces an important decrease in the final length of terminal shoot.

The influence of water deficits also occurs during the following season : the plants which have been grown in drought year-moist year sequence show, during the second year, a greater elongation than that of the plants kept in optimal hydric conditions during the two years. This phenomenon is owed to an earlier budbreak of stressed plants and a stimulation of their rate of growth.

- In the second experiment five provenances of cedar were used. The results obtained in the first one were confirmed. Furthermore the differents provenances show quantitative variations in their reactions to a given water stress. The knowledge of these differences could be used in order to guide the choice of provenances toward the most favourable climatic regions (drought and frost risks) to each of them.

\section{Références bibliographiques}

Aussenac G., 1975. Couverts forestiers et facteurs du climat : leurs intcractions. conséquences écophysiologiques chez quelques résineux. Thèse Dociorat d'Etat, Université Nancy $\mathrm{I}$.

Aussenac G., 1980. Premiers résultats d'une étude de l'influence de l'alimentation en eau sur la croissance des arbres dans un peuplement de douglas. Rev. for. fr., XXXII (2), 167-172.

Aussenac G., Granier A., Gross P., 1980. Etude de la croissance en hauteur du cèdre, utilisation d'un appareillage de mesure automatique. Ann. Sci. for. (sous presse).

Becker M., 1974. Etude expérimentale de la transpiration et du développement de jeunes douglas en fonction de l'alimentation en eau. Ann. Sci. for., 31 (2), 97-110.

Becker M., 1977. Contribution à l'étude de la transpiration et de l'adaptation à la sécheresse des jeunes plants de résineux. Exemple de trois sapins du pourtour méditerranéen : Abies alba, Abies nordmanniana, Abies numidica. Ann. Sci. for., 34 (2), 137-158.

BIDABE B., 1967. Action de la température sur l'évolution des bourgeons de pommier et comparaison de méthodes de contrôle de l'époque de floraison. Ann. Phys. vég., 9 (1), 65-86.

Brochet P., Durand R., Gerbier N., 1971. Recueil de données agroclimatologiques; sommes de températures, monographie $\mathrm{n}^{\prime \prime} 83$ de la Météorologie Nationale, $145 \mathrm{p}$.

Bulletin de la Vulgarisation forestière, 1974a. Le cèdre dans la région Provence-Côte d'Azur, $\mathrm{n}^{\circ}$ 74/06, 33-76.

Bulletin de la Vulgarisation forestière, 1974b. Le reboisement en cèdre dans le Sud-Est de la France, $n^{\circ} 74 / 08,13-58$.

Bulletin de la Vulgarisation forestière, 1979. Le cèdre en Languedoc-Roussillon, $n^{\circ}$ 79/8-9, $11-i 5$.

Clements J.R., 1979.Shoot responses of young red pine to watering applied ower two seasons. Can. J. Bot., 48, 75-80.

KozLowski T.T., 1971. Growth and Development of Tress. Vol. 1, Academic-Press, New York and London, $443 \mathrm{p}$. 
Pradal F., 1979. Variabilité génétique et écophysiologique du cèdre. Mémoire de $3^{e}$ année E.N.I.T.E.F., Nogent-sur-Vernisson, 89 p.

RiedACKer A., 1978. Régénération et croissance de la partie souterraine et aérienne de cèdres placés sous climat constant. Ann. Sci. for., 35 (2), 117-138.

Romberger J.A., 1963. Meristems, growth and development in woody plants. U.S. Department of Agriculture, Forest Service, Technical Bulletin, $\mathrm{n}^{\circ} 1293,214 \mathrm{p}$.

Smith H., Kefford N.P., 1964. The chemical regulation of the dormancy phases of bud development. Am. J. Bot., 51, 1002-1012.

Tотн J., 1971. Notice monographique du cèdre, région méditerranéenne, I.N.R.A. Avignon, $33 \mathrm{p}$.

Tотн J., 1973. Première approche de la production potentielle du cèdre de l'Atlas dans le Sud de la France, Rev. for. fr., XXV (5), 381-389.

VARTANIAN N., 1978a. Influence des facteurs hydriques de l'environnement sur le système racinaire : aspects morphologiques, histologiques et écophysiologiques. Thèse de Doctorat d'Etat, Paris-Orsay, $150 \mathrm{p}$.

Vartanian N., 1978b. Potentialité adaptative du système racinaire à la sécheresse. Bull. Soc. Ecophysiol., 3 (1), 46-47.

YI B.G., 1976. Croissance du cèdre de l'Atlas en relation avec quelques variables du milieu en Languedoc-Roussillon (France). Thèse Docteur-Ingénieur, Montpellier, $133 \mathrm{p}$.

ZAHNER R., 1962. Terminal growth and wood formation by juvenile loblolly pine under two soil moisture regimes. For, Sci., 8, 345-352.

ZAHNER R., 1968. Water deficits and growth of trees, $i n$ "water deficits and plant growth», Tome II (Kozlowski T.T. éd.), 191-254. Academic Press, New York and London.

Zimmermann M.M., Brown C.L., 1971. Trees : Structure and Function, Springer-Verlag, New York, Heidelberg, Berlin, 336 p. 